\title{
Observational Treatment of Internal Thoracic Artery Graft Dissection
}

Keitaro Akita, MD; Hayato Ohtani, MD;

Atsushi Sakamoto, MD; Yuichiro Maekawa, MD
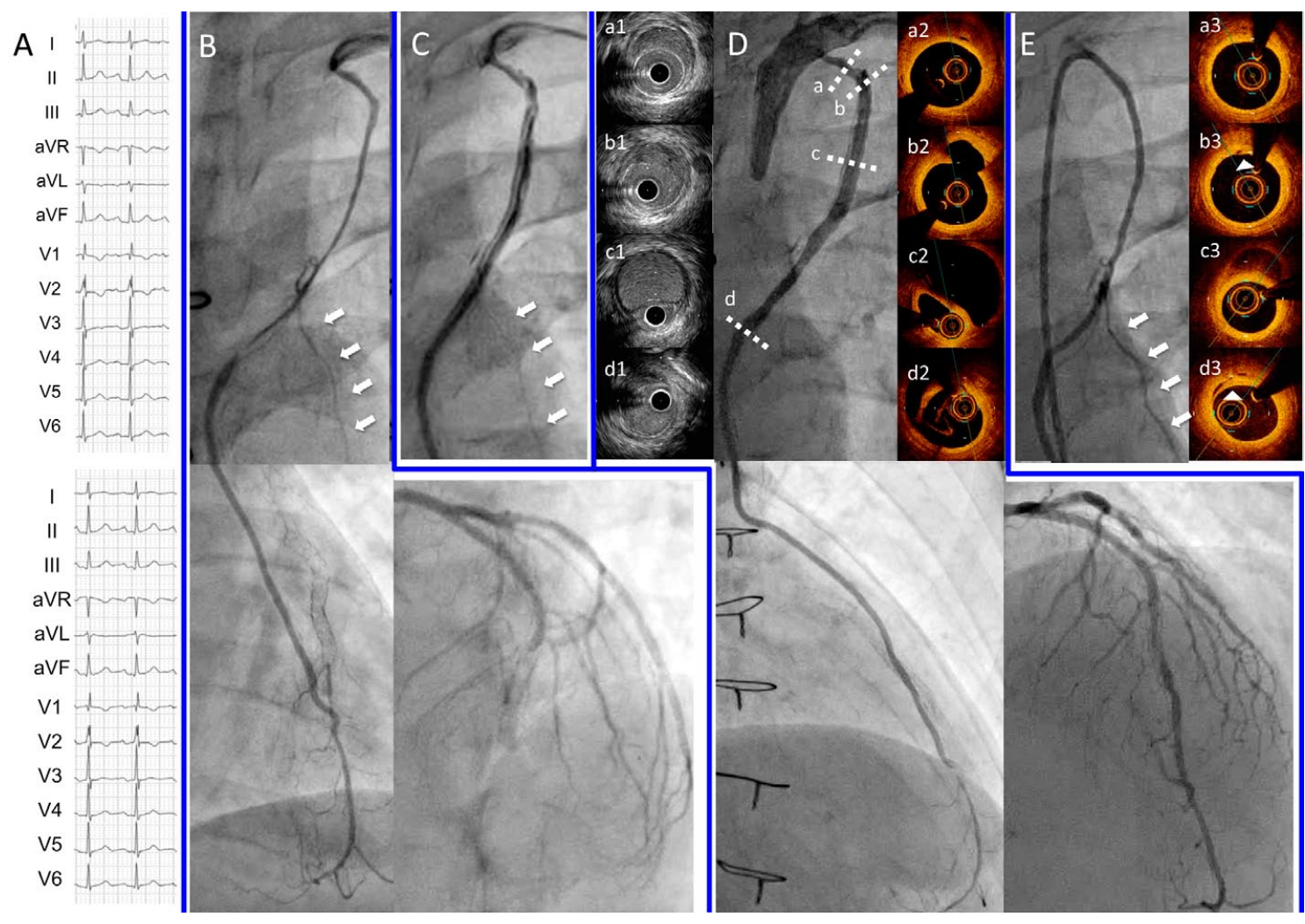

Figure. (A) Electrocardiogram (Top) on admission and (Bottom) after the first angiography. (B) First angiography of the left internal thoracic artery (LITA) and left anterior descending artery (LAD). (C) Second LITA angiography. (D) Third LITA angiography, with intravascular ultrasound (IVUS), optimal coherence tomography (OCT; Supplementary Movie 1) and LAD angiography after percutaneous coronary intervention. (E) LITA angiography 3 months later, with OCT (Supplementary Movie 2). White arrows, LITA side branch. (a1-3) Retrograde dissection. (b1-3) Intimal tear on entry (white arrowhead). (c1-3) Antegrade dissection. (d1-3) Intimal tear on re-entry (white arrowhead).

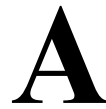
66-year-old man complained of sudden-onset chest pain, continuing for $30 \mathrm{~min}$. He had a history of coronary artery bypass graft with the left internal thoracic artery (LITA) to the left anterior descending (LAD) 3 years earlier, because of refractory in-stent reste- nosis. Due to increased serum troponin I over 2 hours, he underwent emergency coronary angiogram, which showed in-stent occlusion in the LAD and $99 \%$ stenosis in the LITA (Figure B). Other coronary arteries were normal. Given that there had been neither chest pain nor ischemic

Received October 9, 2018; revised manuscript received July 22, 2019; accepted August 13, 2019; J-STAGE Advance Publication released online September 14, 2019 Time for primary review: 80 days

Internal Medicine III, Hamamatsu University School of Medicine, Hamamatsu, Japan

Mailing address: Keitaro Akita, MD, Internal Medicine III, Hamamatsu University School of Medicine, 1-20-1 Handayama, Higashi-ku, Hamamatsu 431-3192, Japan. E-mail: akita@hama-med.ac.jp

ISSN-2434-0790 All rights are reserved to the Japanese Circulation Society. For permissions, please e-mail: cr@j-circ.or.jp 
ST-T changes (Figure A), we observed him for 1 week. The second angiogram showed a double-barreled LITA, implying dissection (Figure C). After another week of observation, the third angiogram showed that a side branch of the LITA had disappeared, which implied the false lumen had become dominant (Figure D). Given that the long-term patency of the LITA was not guaranteed, revascularization for either LAD or LITA seemed necessary. We performed an intervention on the LAD with a drug-coated balloon, then observed LITA on intravascular ultrasound (IVUS) and optimal coherence tomography (OCT; Figure D; Supplementary Movie 1). Intimal tears were observed in the entry and re-entry site of dissection. The chest pain never relapsed after the treatment. Three months later, angiogram showed that the LAD was patent, and the LITA recovered to a single-barreled state (Figure E). On OCT, intimal tears were totally healed (Figure E; Supplementary Movie 2).

Whereas previous reports of ITA dissections documented stent implantation treatment, ${ }^{\mathbf{1}}$ the dissected LITA in the present case was carefully observed as it gradually healed. The recovered flow of the distal LAD may have promoted the degeneration of the false lumen of the LITA via anastomosis. This report has described natural healing after ITA dissection, implying the possibility of observational treatment.

\section{Disclosures}

None.

\section{Reference}

1. Freixa $X$, Gallo R. Internal thoracic artery dissection: A proposed mechanistic explanation. JACC Cardiovasc Interv 2013; 6: $533-$ 534.

\section{Supplementary Files}

Supplementary Movie 1. Optical coherence tomography of the dissected LITA.

Supplementary Movie 2. Optical coherence tomography of the healed LITA.

Please find supplementary file(s);

http://dx.doi.org/10.1253/circrep.CR-18-0032 